MATHEMATICS OF COMPUTATION

Volume 80, Number 276, October 2011, Pages 2435-2444

S $0025-5718(2011) 02461-0$

Article electronically published on January 20, 2011

\title{
IRREGULAR PRIMES TO 163 MILLION
}

\author{
J. P. BUHLER AND D. HARVEY
}

\begin{abstract}
We compute all irregular primes less than 163577856 . For all of these primes we verify that the Kummer-Vandiver conjecture holds and that the $\lambda$-invariant is equal to the index of irregularity.
\end{abstract}

\section{INTRODUCTION}

Bernoulli numbers are deeply intertwined with the arithmetic of cyclotomic fields. To explain a basic case, consider the cyclotomic field $K=\mathbf{Q}\left(\zeta_{p}\right)$ generated by a $p$-th root of unity $\zeta_{p}=e^{2 \pi i / p}, p$ prime. Let $G=\operatorname{Gal}(K / \mathbf{Q}) \simeq(\mathbf{Z} / p \mathbf{Z})^{\times}$be its Galois group, and let $\omega: G \rightarrow \mathbf{Z}_{p}^{\times}$be the unique $p$-adic character for which

$$
\omega(\sigma) \equiv r \bmod p
$$

where $\sigma\left(\zeta_{p}\right)=\zeta_{p}^{r}$. Then the $p$-Sylow subgroup $A$ of the class group decomposes into components

$$
A=\bigoplus_{0<k<p-1} A_{k}
$$

where $A_{k}$ is the $\mathbf{Z}_{p}[G]$-submodule on which $\sigma$ acts by multiplication by $\omega^{k}(\sigma)$. (For details, see Was97.)

It is conjectured that $A_{k}$ is trivial if $k$ is even; this is equivalent to the Vandiver (or Kummer-Vandiver) conjecture that the class number $h_{p}^{+}$of the maximal real subfield $\mathbf{Q}\left(\zeta_{p}+\zeta_{p}^{-1}\right)$ is not divisible by $p$. By combining results of Herbrand and Ribet we know that $A_{p-k}$ is nontrivial if and only if the numerator of the $k$-th Bernoulli number is divisible by $p$, and the Main Conjecture, proved by Mazur and Wiles, shows that the order of $A_{p-k}$ is equal to the power of $p$ that divides $B_{k}$. Thus, the first step in finding the $p$-part of the class group of $K$ is to study the numerators of Bernoulli numbers.

If $k$ is an even integer with $0<k<p-1$, then $k$ is said to be an irregular index for $p$ if $p$ divides the numerator of the $k$-th Bernoulli number $B_{k}$. The index of irregularity of $p$, denoted $i_{p}$, is the number of such irregular indices $k$, and $p$ is irregular if $i_{p}$ is positive. If $k$ is irregular for $p$, then $(p, k)$ is said to be an irregular pair. Once the irregular pairs for a given $p$ have been found it is possible to make further calculations verifying the Kummer-Vandiver conjecture, and to calculate the corresponding $\lambda$-invariants in Iwasawa theory; these invariants $\lambda_{p}$ measure the rate of growth of the $p$-part of the class group of $p$-power cyclotomic fields.

This paper describes a calculation of all irregular pairs for $p$ less than $39 \cdot 2^{22}=$ 163577856 . The computation consumed more than twenty years of CPU time,

Received by the editor March 3, 2010 and, in revised form, June 18, 2010

2010 Mathematics Subject Classification. Primary 11Y40; Secondary 11R18.

(c) 2011 by the authors 
and the quick summary of the results is that nothing surprising happened. The Kummer-Vandiver conjecture is true for all of these primes, and the $\lambda$-invariant is always as small as it can be, i.e., $\lambda_{p}=i_{p}$. This implies that the $p$-part of the class group of $\mathbf{Q}\left(\zeta_{p^{n}}\right)$ is, for all of these $p$, isomorphic to $\left(\mathbf{Z} / p^{n} \mathbf{Z}\right)^{i_{p}}$. We did find one new prime with $i_{p}=7$, namely $p=32012327$. At the time we wrote this, the data supporting these calculations could be found at Har09c.

Irregular indices have been computed many times over the last 160 years, starting with Kummer's hand calculations for $p \leq 163$, which he used to verify Fermat's Last Theorem and that $h_{p}^{+}$is prime to $p$. This was extended to $p \leq 619$ early in the twentieth century by Vandiver and his colleagues, using desk calculators (and graduate students). Vandiver and the Lehmers, making one of the first numbertheoretic calculations on electronic computers, extended this to $p \leq 4001$ by 1956 . For a discussion of these early results, see Cor08. A series of further results by the Lehmers, Iwasawa, Sims, Kobelev, Johnson, Nicol, Pollack, Selfridge, Tanner, and Wagstaff extended these to $p<150000$ by 1987 (see [Joh75, Wag78, or [TW87] for a summary and further references). In the early 1990s, Buhler, Crandall, Ernvall, Metsänkylä, Sompolski, and Shokrollahi used "asymptotically fast" algorithms to go much further, and by 1999 the computations were extended to all primes less than 12 million BCS92, BCEM93, BCE01, EM91, EM92.

This paper describes our extension of these results. The two major reasons that we were able to extend the upper bound on $p$ by a factor of almost 14 were: (a) the use of two large clusters at the Texas Advanced Computing Center at the University of Texas at Austin, and (b) new algorithms and implementations of them, due to the second author, for polynomial arithmetic over finite fields.

The paper is organized as follows: we first describe the algorithms for calculating $B_{k} \bmod p$ for $0<k<p-1$, algorithms for performing the needed polynomial arithmetic, and the algorithms for the Vandiver and cyclotomic invariant calculations. Then we summarize the results, including a description of the hardware that we used and a discussion of the steps we took to ensure correctness.

\section{Bernoulli numbers modulo $p$}

Bernoulli numbers are defined by a formal power series inversion

$$
\frac{t}{e^{t}-1}=\sum_{k \geq 0} B_{k} \frac{t^{k}}{k !} .
$$

We have $B_{1}=1 / 2$, and all other odd Bernoulli numbers $B_{3}, B_{5}, \ldots$ are zero. The first, and by far and away the most time-consuming phase of our calculations is the computation of $B_{k}$ mod $p, 0 \leq k<p-1, k$ even, for each $p$ in turn.

We used two different algorithms, the Voronoi identity method and the power series method. Both depend crucially on asymptotically fast polynomial arithmetic over $\mathbf{Z} / p \mathbf{Z}$. The theoretical complexity is $O\left(p \log ^{2+\varepsilon} p\right)$ for both methods, but the implied constants and memory footprints are different - the algorithm using the Voronoi identity seems to be faster but requires more memory. We first describe the two methods, and then discuss the algorithms used for the underlying polynomial arithmetic.

The method based on the Voronoi identity is essentially identical to the "root finding method" of BCE01; we used it for all $p<21 \cdot 2^{22}=88080384$. Let $g$ be 
a generator of $(\mathbf{Z} / p \mathbf{Z})^{\times}$. Then for $2 \leq k<p-1, k$ even, we have

$$
B_{k} \equiv \frac{2 k}{1-g^{k}} \sum_{i=0}^{(p-3) / 2} g^{(k-1) i} h\left(g^{i}\right) \quad(\bmod p),
$$

where

$$
h(x)=\frac{(x \bmod p)-g(x / g \bmod p)}{p}+\frac{g-1}{2} .
$$

This is a variation, with the terms arranged in multiplicative order, of a congruence due to Voronoi. This was published in Comm. Chakov Math. Soc. in 1890, and also appeared in his collected works; numerous closely related results can be found in the literature; see [Sla87, Van17] for examples, or DSS91] for an extensive Bernoulli number bibliography (updated online version at Dil09]). The sum on the right-hand side may be regarded as the Fourier transform of $i \mapsto h\left(g^{i}\right) / g^{i}$ with respect to the roots of unity $\left\{g^{2 k}\right\}_{k=0}^{(p-3) / 2}$. Using Bluestein's FFT algorithm Blu70, we convert the problem of simultaneously computing all $B_{k}$ to the problem of multiplying two polynomials of length $(p-1) / 2$ over $\mathbf{Z} / p \mathbf{Z}$, plus $O(p)$ pre-processing and postprocessing operations in $\mathbf{Z} / p \mathbf{Z}$.

The power series method is described in BCS92]. We used the variant based on the identity

$$
\sum_{n=0}^{\infty} \frac{2^{n} B_{2 n}}{(2 n) !} x^{2 n}=\frac{A_{0}\left(x^{8}\right)+A_{2}\left(x^{8}\right) x^{2}+A_{4}\left(x^{8}\right) x^{4}+A_{6}\left(x^{8}\right) x^{6}}{D\left(x^{8}\right)},
$$

where $D$ and the $A_{i}$ are power series whose coefficients are given by simple recurrence formulae [BCS92, p. 719]. This reduces to a power series inversion of length $p / 8+O(1)$, and four series multiplications of length $p / 8+O(1)$, over $\mathbf{Z} / p \mathbf{Z}$. This method uses less memory than the Voronoi identity method, and we used it for all primes $21 \cdot 2^{22}<p<39 \cdot 2^{22}$.

\section{Polynomial and Power series ARithmetic}

Most of the algorithms for polynomial and power series arithmetic described here are implemented in the zn_poly library of the second author [Har08b].

In what follows, we consider arithmetic in $R[x]$, including arithmetic on truncated power series in $R \llbracket x \rrbracket$, where $R$ is a coefficient ring in which 2 is not a zero-divisor. For the algorithms described above, we could take $R=\mathbf{Z} / p \mathbf{Z}$, where $p$ is the prime of interest. In our implementation, we found it profitable to handle two nearby primes simultaneously, taking instead $R=\mathbf{Z} / p_{1} p_{2} \mathbf{Z}$, and using the Chinese Remainder Theorem. This was possible since the computations were run on 64-bit hardware, and the largest prime considered was less than 32 bits long.

Our large polynomial multiplication routine involves a combination of algorithms. The outermost layer reduces the multiplication of polynomials $f, g \in$ $R[x]$ of length $n$ to $O\left(n^{1 / 2}\right)$ negacyclic convolutions of length $O\left(n^{1 / 2}\right)$, using an adaptation to polynomials of the Schönhage-Strassen integer multiplication algorithm [SS71] suggested by Schönhage [Sch77]. This proceeds by splitting the inputs into segments of length $M / 2$ and embedding the multiplication problem into $R[y, z] /\left(y^{M}+1, z^{K}-1\right)$ via the inverse of the substitution $y \mapsto x, z \mapsto x^{M / 2}$, where $M=2^{\left\lceil\left(1+\log _{2} n\right) / 2\right\rceil}=O\left(n^{1 / 2}\right)$ and $K=2^{\left\lceil\log _{2}(4 n / M)\right\rceil}=O\left(n^{1 / 2}\right)$. These conditions ensure that $M K \geq 4 n$ (the product "fits" into the bivariate quotient ring) and that $K \mid 2 M$. The bivariate multiplication may then be effected by using FFTs over the 
ring $R[y] /\left(y^{M}+1\right)$ with respect to the primitive $K$-th root of unity $y^{2 M / K}$. These FFTs involve only additions and subtractions in $R$, plus some bookkeeping to keep track of powers of $y$. To improve memory locality and smoothness of the running time with respect to $n$, we use a cache-friendly version Har09a of van der Hoeven's truncated Fourier transform (TFT) method vdH04, vdH05.

For the negacyclic convolutions (recursive multiplications in $R[y] /\left(y^{M}+1\right)$ ) we use Nussbaumer's algorithm Nus80 for sufficiently large $M$; this follows the same general plan as Schönhage's algorithm sketched above, but uses a slightly different substitution that can reduce the size of the Fourier coefficients by a factor of two in some cases. For sufficiently small $M$, we switch to a variant of the Kronecker substitution method [Har09b], which packs the input polynomials into integers and multiplies the resulting integers.

Our power series inversion algorithm is based on a Newton iteration. We followed the approach suggested in HQZ04, Algorithm MP-inv, p. 421], which takes advantage of the middle product to improve the running time constant. A minor improvement in our algorithm is that we reuse the Fourier transform of $\alpha$ in lines 3 and 4 of HQZ04, reducing the theoretical cost by a further $1 / 6$. To transport the smoothness benefits of the truncated FFT from the ordinary product to the middle product, it was necessary to implement a transposed TFT and inverse TFT over $R[y] /\left(y^{M}+1\right)$, making use of the "transposition principle" BLS03. Unlike the usual (nontruncated) FFT, the matrices for the TFT and inverse TFT are not symmetric, so genuinely new code was required.

It is worth pointing out that our polynomial arithmetic routines are based entirely on integer arithmetic; we did not use floating point arithmetic at all.

Memory constraints became formidable for those $p$ near the top of the range that we handled using the Voronoi identity method. Just to store two polynomials of length $p / 2$ and their product, where $p \approx 21 \cdot 2^{22}$, requires 1.4 GB of memory, assuming a 64 -bit word for each coefficient. Each thread had only 2 GB RAM available (see description of the hardware below), leaving very little auxiliary working storage, certainly much less than is required to store the FFT coefficients. To overcome this, we implemented a discrete Fourier transform variant that uses a naive quadratic-time DFT algorithm to handle the first few layers of the transform, and then the standard $O(n \log n)$ algorithm for the remaining layers. The resulting time-space tradeoff was acceptable up to some limit, but became infeasible as we approached the $2 \mathrm{~GB}$ barrier, prompting us to switch to the less memory-intensive power series method.

\section{KummeR-VANDIVER AND CYCLOTOMIC INVARIANTS}

We quickly summarize some well-known facts about cyclotomic class groups, using notation established above; for details see Was97. If $p$ is prime and $k$ is even, $0 \leq k<p-1$, then $A_{p-k}$ is nontrivial if and only if $k$ is irregular for $p$. Moreover, a "reflection theorem" implies that if $A_{k}$ is nontrivial, then $A_{p-k}$ is nontrivial, i.e., $k$ is irregular for $p$. The component $A_{k}$ has the same order as the $\omega^{k}$-component of the $p$-Sylow subgroup of $U_{K} / \mathrm{Cyc}_{K}$, where $U_{K}=O_{K}^{\times}$is the group of units inside the ring of integers of $K$, and $\mathrm{Cyc}_{K}$ denotes the group of cyclotomic units. The latter can be described explicitly, and Vandiver's conjecture for $p$, which says that $A_{k}$ is trivial for all even $k$, can be proved by showing that the cyclotomic unit $u_{k}$ that generates this component does not have a $p$-th root lying in $K$. 
This can be established by finding a prime ideal $Q$ of $K$ that has residue class degree 1 , for which $u_{k}$ is not a $p$-th power modulo $Q$. This reduces (again, for details see Was97]) to showing that it is possible to find a rational prime $q$ with $q \equiv 1 \bmod p$ such that $V_{p, k}^{(q-1) / p} \not \equiv 1 \bmod q$, where

$$
V_{p, k}=\prod_{c=1}^{(p-1) / 2}\left(z^{c}-z^{-c}\right)^{c^{p-1-k}}(\bmod q) .
$$

Here $z$ is a $p$-th root of unity modulo $q$, and $Q$ is one of the primes lying over $q$. Thus, Vandiver's conjecture for $p$ is proved if the above condition is checked for each irregular index $k$ for $p$. We performed this computation for every irregular pair in our table, and in each case the above condition held, and in all cases the smallest prime $q \equiv 1 \bmod p$ worked.

To verify that the $\lambda$-invariant $\lambda_{p}$ is equal to $i_{p}$ it suffices to check that certain congruences between Bernoulli numbers do not hold. These reduce to elementary congruences [EM91. To describe these, let

$$
S(e)=\sum_{a=1}^{(p-1) / 2} a^{e} .
$$

For each irregular index $k$ for a given prime $p$ we check that

$$
\begin{aligned}
2^{k} & \not \equiv \bmod p, \\
S(k-1) & \neq S(p+k-2) \bmod p^{2}, \\
k S(k-1) & \not \equiv(k-1) S(p+k-2) \bmod p^{2} .
\end{aligned}
$$

As explained in EM91 these imply that (the numerator of) $B_{k}$ is not divisible by $p^{2}$, that the generalized Bernoulli number $B_{1}\left(\omega^{k-1}\right)$ is not divisible by $p^{2}$ and that

$$
\frac{B_{k}}{k} \not \equiv \frac{B_{k+p-1}}{k+p-1} \bmod p^{2} \text {. }
$$

If these conditions hold for all irregular indices $k$ for $p$, then $\lambda_{p}=i_{p}$. In the case that $2^{k} \equiv 1 \bmod p$, this test can be replaced by another one EM91.

\section{Results}

There are $N=9163831$ primes up to $39 \cdot 2^{22}=163577856$. The indices of irregularity $i_{p}$ ranged up to 7 , and the number of primes $N_{i}$ of index $i$ are tabulated below.

Here $p_{i}=e^{-1 / 2} /\left(2^{i} i\right.$ !) is the probability that a Poisson process with mean $1 / 2$ has $i$ successes. This is motivated by the heuristic (Lehmer, Siegel) that if the Bernoulli numerators are random modulo $p$, then $i_{p}$ is the number of successes in $p / 2$ trials each having probability $1 / p$ of success.

The five primes of index 7 are $3238481,5216111,5620861,9208289$ and 32012327.

The Vandiver conjecture was found to be true for all primes tested. The $\lambda$ invariant was equal to $i_{p}$ for all primes; the alternate test mentioned above was required for 3 primes. 
TABLE 1. Irregular index statistics for $p<39 \cdot 2^{22}$

\begin{tabular}{rrllr}
\hline$i$ & $N_{i}$ & $N_{i} / N$ & $p_{i}$ & $N \cdot p_{i}$ \\
\hline 0 & 5559267 & 0.6067 & 0.6065 & 5558144 \\
1 & 2779293 & 0.3033 & 0.3032 & 2779072 \\
2 & 694218 & 0.0758 & 0.0758 & 694768 \\
3 & 115060 & 0.01256 & 0.01263 & 115794 \\
4 & 14425 & 0.00157 & 0.00158 & 14474 \\
5 & 1451 & 0.000158 & 0.000158 & 1447 \\
6 & 112 & 0.000012 & 0.000013 & 120 \\
7 & 5 & 0.00000055 & 0.00000094 & 8 \\
\hline
\end{tabular}

For a discussion of heuristics that might apply to Vandiver's conjecture and to the value of $\lambda_{p}$ see Was97, p. 159] and [Lan90, p. 261]. If $(p, k)$ is an irregular pair, Washington examines the consequences of assuming that the probability that the cyclotomic unit $u_{k}$ is a $p$-th power is $1 / p$, all independently of each other. Using this and heuristics on the distribution of $i_{p}$, he guesses that the number of counterexamples to Vandiver's conjecture for $p<x$ should grow like $\frac{1}{2} \log \log x$. As an exercise in futility, we may try to refine Washington's heuristics using the known values of $i_{p}$, taking into account for example that the first irregular prime $(p=37)$ is unexpectedly large. We find that the expected number of counterexamples up to 12 million is about 0.674 , and that another 0.074 counterexamples were expected between 12 million and 163 million (though of course we now know that there are none in either case). Many people believe that Vandiver's conjecture is true; it also seems reasonable to believe that the conjecture is false but that the first counterexample is so astronomically large that it may never be known. Similar remarks apply to the $\lambda_{p}=i_{p}$ conjecture.

As mentioned earlier, the table of irregular pairs, together with check data for the Vandiver and cyclotomic invariant verifications, can be found at Har09c].

\section{Correctness}

Any computation on this scale is virtually guaranteed to encounter faults of various kinds, and this computation was no exception. On one occasion during the main Bernoulli number computation, a hardware or operating system failure - we could not determine which - resulted in several kilobytes of output being overwritten by random data, and it was necessary to repeat the computation for the affected primes. On another occasion, the Vandiver and cyclotomic runs located subtle memory errors (subsequently confirmed by other tests) on two workstations. In comparing our data for those tests, we found that a handful of examples (which were recalculated) were some sort of write error invalidating a very small number of lines. To combat this sort of occurrence, we took careful precautionary measures throughout the project, which we now describe.

The main irregular index computation was far too expensive to run more than once. We checked the correctness of this phase by several methods. During the 
main computation itself, for each $p$ we verified the identity

$$
\sum_{k=0}^{p-3} 2^{k}(k+1) B_{k} \equiv-4 \quad(\bmod p)
$$

(see BCS92, p. 720]). Already this is a strong consistency check, but it has the drawback that it leaves no certificate that can be checked after the computation is finished. To increase our confidence in the output, we made our program record somewhat more than just the irregular indices. Let $N_{p}=\min \left(2 \log p, \frac{1}{2}(p-3)\right)$. After computing all $B_{k}$ mod $p$ for a given $p$, we extracted and stored the $N_{p}$ smallest pairs $\left(k, B_{k} \bmod p\right)$, where the pairs are ordered first by $B_{k} \bmod p$ and then by $k$. In particular, the irregular pairs are listed first, and for the largest primes considered we store about 37 pairs. The resulting compressed file is $1.5 \mathrm{~GB}$, and is available for download Har09c. We then used a completely independent program and hardware to check these recorded pairs. For each $k$, one may compute $B_{k} \bmod p$ in $O(p)$ operations using an identity such as (1). We used the highly optimized implementation described in Har08a.

This scheme has an additional property we might expect of a "certificate". A skeptic may randomly select and verify a pair $\left(k, B_{k} \bmod p\right)$ from our output file. Since presumably the only way to find a $k$ for which $B_{k} \bmod p$ is "small" is to compute all the $B_{k}$ mod $p$, the skeptic may be persuaded that we must have actually performed the full computation for each $p$. Note that recording all the $B_{k} \bmod p$ that we computed is impractical, requiring in the order of $10^{6} \mathrm{~GB}$ of storage.

For the Vandiver tests, we computed $V_{p, k}$ twice for each irregular pair and crosschecked the results. This was done using programs written independently by the two authors, working separately and sharing no code. The programs were run on different hardware, using different libraries for the modular arithmetic (zn_poly and NTL Sho09]).

The first run used a new implementation of the method described in BCE01, updated for 64-bit hardware. For the second run, we used a slightly different algorithm. Instead of iterating over $c$ from 1 up to $(p-1) / 2$, we write $c=2^{i} g^{j}$, where $g$ is a primitive root modulo $p$, and iterate over $0 \leq i<t$ in an inner loop and $0 \leq j<(p-1) / t$ in an outer loop, where $t$ is the order of 2 modulo $p$. For each pair $(i, j)$ we only include the multiplicand in the product if the proposed $c$ satisfies $1 \leq c \leq(p-1) / 2$. The advantage of this approach is that only a single multiplication in $\mathbf{Z} / p \mathbf{Z}$ is required to update $c^{p-1-k}$ on each iteration, rather than the $O(\log p)$ operations needed to compute each $c^{p-1-k}$ had the $c$ been processed sequentially. Updating $z^{c}$ under this scheme requires only a single operation in the inner loop (since $z^{2^{i+1} g^{j}}=\left(z^{2^{i} g^{j}}\right)^{2}$ ), and slightly more in the outer loop. The latter executes infrequently for most $p$, since $t$ is usually large.

For the cyclotomic invariants, we again ran everything twice, using independent programs on different hardware. The first run used the implementation from BCE01, and the second was written from scratch using NTL's modular arithmetic.

Earlier computations of irregular pairs and associated data uncovered minor problems in their predecessors' data, so it was natural for us to compare our data with the results up to 12 million from ten years ago. We found four errors in the online tables, and are confident that three of the cases were introduced by an illadvised editing, by hand, of the table to correct cases in which a faulty computer had been used, and one of the cases was output of the faulty computer itself. 


\section{HARDWARE}

The aforementioned computations were performed on a number of different machines:

"Lonestar" is a 1300-node cluster at the Texas Advanced Computing Center (TACC), running a custom Linux operating system. Each node contains two dualcore $2.66 \mathrm{GHz}$ Intel Xeon (Woodcrest) processors and has 8 GB RAM. This machine was used for computing irregular indices (about 119000 core-hours). We used various versions of GMP (4.2.1-4.2.4) Gra08 for the large integer multiplication, patched with assembly code written by Jason Martin for the Core 2 architecture.

"Ranger" is a 3936-node cluster at TACC, running a custom Linux operating system. Each node contains four quad-core 2.3 GHz AMD Opteron (Barcelona) processors and has 32 GB RAM. At the time the computations were run, this machine was ranked by top500.org as the fourth most powerful supercomputer in the world. This machine was used for computing irregular indices (about 68000 core-hours). We used the same versions of GMP mentioned above, together with assembly code written by the second author and Torbjörn Granlund. This code has subsequently been incorporated into public releases of GMP; indeed, the needs of the present computation were a large part of the motivation for this work on GMP.

The first run of the Vandiver and cyclotomic invariant checks was performed on a network of 24 desktop machines, each with a $3.4 \mathrm{GHz}$ Pentium $4 \mathrm{CPU}$ (about 16000 core-hours). The second run was performed on a 16-core $2.6 \mathrm{GHz}$ Opteron server (about 10000 core-hours). This machine was also used for the verification of the $B_{k} \bmod p$ (about 500 core-hours).

\section{ACKNOWLEDGEMENTS}

Many thanks to Torbjörn Granlund for contributing his invaluable assembly programming expertise.

The authors acknowledge the Texas Advanced Computing Center (TACC) at The University of Texas at Austin for providing high-performance computing resources that have contributed to the research results reported within this paper.

Thanks to Fernando Rodriguez Villegas and the Department of Mathematics at the University of Texas at Austin for arranging access to the clusters at TACC, and to Salman Butt for his technical assistance with the clusters.

Thanks to the Department of Mathematics at Harvard University for supplying the Opteron server.

The first author thanks Richard Crandall and Amin Shokrollahi for useful conversations on this paper.

\section{REFERENCES}

[BCE01] J. Buhler, R. Crandall, R. Ernvall, T. Metsänkylä, and M. Shokrollahi, Irregular primes and cyclotomic invariants to 12 million, J. Symbolic Comput. 31 (2001), no. 1-2, 89-96, Computational algebra and number theory (Milwaukee, WI, 1996). MR:1806208 (2001m:11220)

[BCEM93] J. Buhler, R. Crandall, R. Ernvall, and T. Metsänkylä, Irregular primes and cyclotomic invariants to four million, Math. Comp. 61 (1993), no. 203, 151-153. MR.1197511 (93k:11014)

[BCS92] J. P. Buhler, R. E. Crandall, and R. W. Sompolski, Irregular primes to one million, Math. Comp. 59 (1992), no. 200, 717-722. MR1134717 (93a:11106) 
[BLS03] A. Bostan, G. Lecerf, and É. Schost, Tellegen's principle into practice, Symbolic and Algebraic Computation (J. R. Sendra, ed.), ACM Press, 2003, Proceedings of ISSAC'03, Philadelphia, August 2003., pp. 37-44.

[Blu70] L. Bluestein, A linear filtering approach to the computation of discrete Fourier transform, Audio and Electroacoustics, IEEE Transactions on 18 (1970), no. 4, 451-455.

[Cor08] L. Corry, Fermat meets SWAC: Vandiver, the Lehmers, computers, and number theory, IEEE Ann. Hist. Comput. 30 (2008), no. 1, 38-49. MR2412202 (2009b:11002)

[Dil09] 2009, http://www.mscs.dal.ca/ ${ }^{\text {dilcher/bernoulli.html/. }}$

[DSS91] K. Dilcher, L. Skula, and I. Slavutskiǐ, Bernoulli numbers, Queen's Papers in Pure and Applied Mathematics, vol. 87, Queen's University, Kingston, ON, 1991, Bibliography (1713-1990). MR1119305 (92f:11001)

[EM91] R. Ernvall and T. Metsänkylä, Cyclotomic invariants for primes between 125000 and 150000, Math. Comp. 56 (1991), 851-858. MR.1068819 (91h:11157)

[EM92] - Cyclotomic invariants for primes up to one million, Math. Comp. 59 (1992), 249-250. MR1134727 (93a:11108)

[Gra08] T. Granlund, The GNU Multiple Precision Arithmetic library, 2008, http://gmplib.org/.

[Har08a] D. Harvey, A multimodular algorithm for computing Bernoulli numbers, Math. Comp. 79 (2010), 2361-2370

[Har08b] _ The zn_poly library, 2008, http://www.cims.nyu.edu/ ${ }^{\sim}$ harvey/zn_poly/.

[Har09a] A cache-friendly truncated FFT, Theoret. Comput. Sci. 410 (2009), no. 27-29, 2649-2658. MR2531107 (2010g:68327)

[Har09b] , Faster polynomial multiplication via multipoint Kronecker substitution, J. Symbolic Comput. 44 (2009), no. 10, 1502-1510. MR2543433

[Har09c] , http://www.cims.nyu.edu/ ${ }^{\sim}$ harvey/irregular/, 2009.

[HQZ04] G. Hanrot, M. Quercia, and P. Zimmermann, The middle product algorithm, I., Appl. Algebra Engrg. Comm. Comput. 14 (2004), no. 6, 415-438. MR2042800(2005a:65003)

[Joh75] W. Johnson, Irregular primes and cyclotomic invariants, Math. Comp. 29 (1975), 113-120. MR0376606 (51:12781)

[Lan90] S. Lang, Cyclotomic fields I and II, Graduate Texts in Mathematics, vol. 21, SpringerVerlag, New York, 1990. MR1029028 (91c:11001)

[Nus80] H. Nussbaumer, Fast polynomial transform algorithms for digital convolution, IEEE Trans. Acoust. Speech Signal Process. 28 (1980), no. 2, 205-215. MR563380 (80m:94004)

[Sch77] A. Schönhage, Schnelle Multiplikation von Polynomen über Körpern der Charakteristik 2, Acta Informat. 7 (1976/77), no. 4, 395-398. MR0436663 (55:9604)

[Sho09] V. Shoup, NTL: A library for doing number theory, http://www.shoup.net/ntl/, 2009.

[Sla87] I. Sh. Slavutskir, A remark on the paper of T. Uehara: "On p-adic continuous functions determined by the Euler numbers" [Rep. Fac. Sci. Engrg. Saga Univ. Math. No. 8 (1980), 1-8; MR0567622 (81e:12020)], Rep. Fac. Sci. Engrg. Saga Univ. Math. 15 (1987), 1-2. MR879252 (88c:11071)

[SS71] A. Schönhage and V. Strassen, Schnelle Multiplikation grosser Zahlen, Computing (Arch. Elektron. Rechnen) 7 (1971), 281-292. MR0292344 (45:1431)

[TW87] J. Tanner and S. Wagstaff, New congruences for the Bernoulli numbers, Math. Comp. 48 (1987), 341-350. MR866120 (87m:11017)

[Van17] H. S. Vandiver, Symmetric functions formed by systems of elements of a finite algebra and their connection with Fermat's quotient and Bernoulli numbers, Ann. Math. 18 (1917), 105-114. MR 1503591

[vdH04] J. van der Hoeven, The truncated Fourier transform and applications, ISSAC 2004, ACM, New York, 2004, pp. 290-296. MR2126956

[vdH05] , Notes on the truncated Fourier transform, unpublished, available from http://www.math.u-psud.fr/ vdhoeven/, 2005.

[Wag78] S. Wagstaff, The irregular primes to 125000, Math. Comp. 32 (1978), 583-591. MR0491465 (58:10711)

[Was97] L. Washington, Introduction to cyclotomic fields, second ed., Graduate Texts in Mathematics, vol. 83, Springer-Verlag, New York, 1997. MR1421575 (97h:11130) 
Center for Communications Research, 4230 Westerra Ct., San Diego, California 92121

E-mail address: buhler@ccrwest.org

Courant Institute of Mathematical Sciences, New York University, 251 Mercer St, New York, New York 10012

E-mail address: dmharvey@cims.nyu.edu 